\title{
Rapid hydrothermal route to synthesize cubic-phase gadolinium oxide nanorods
}

\author{
SAMIRAN HAZARIKA, NIBEDITA PAUL and DAMBARUDHAR MOHANTA* \\ Department of Physics, Tezpur University, Tezpur 784 028, India
}

MS received 21 July 2013; revised 12 September 2013

\begin{abstract}
An inexpensive fabrication route and growth mechanism is being reported for obtaining quality gadolinium oxide $\left(\mathrm{Gd}_{2} \mathrm{O}_{3}\right)$ nanoscale rods. The elongated nanoscale systems, as produced via a hydrothermal process, were characterized by $\mathrm{X}$-ray diffraction (XRD), high resolution transmission electron microscopy (HRTEM), optical absorption spectroscopy, photoluminescence (PL) spectroscopy, Raman spectroscopy and magnetic hysteresis measurements. XRD patterns of the nanorods, as-prepared from independent precursors of different $\mathbf{p H}$, depict a cubic crystal phase and an average crystallite size of 5-6.5 $\mathbf{~ m m}$. As revealed from HRTEM micrographs, diameter of the nanorods prepared at $\mathbf{p H}=13.3(\sim 7 \mathrm{~nm})$ was much smaller than the rods prepared at $\mathrm{pH}=10.8(\sim 19 \mathrm{~nm})$. However, the aspect ratio was more than double in the former case than the latter case. PL response was found to be dominated by defect mediated emissions, whereas Raman spectrum of a given specimen $(\mathrm{pH}=10 \cdot 8)$ has revealed characteristic $F_{\mathrm{g}}+A_{\mathrm{g}}$ modes of cubic phase of $\mathrm{Gd}_{2} \mathrm{O}_{3}$ nanorods, apart from other independent modes. Furthermore, $M \sim H$ plot of the nanorod system $(\mathrm{pH}=10.8)$ exhibited slight departure from the ideal superparamagnetic behaviour, with low remanence and coercive field values. The exploitation of one-dimensional $\mathrm{Gd}_{2} \mathrm{O}_{3}$ nanorods have immense potential in the production of advanced contrast agents, smart drives and also in making novel ferrofluids of technological relevance.
\end{abstract}

Keywords. Hydrothermal; nanostructure; rare-earth; gadolinium oxide.

\section{Introduction}

In recent years, compared to bulk and other low dimensional structures, elongated nanostructures such as nanowires, nanorods and nanotubes have gained remarkable research interest owing to their novel electrical, optical and magnetic properties (Du and Tendeloo 2005). Organized, but anisotropic growth of one-dimensional (1-D) systems, under specific environment, has emerged as an area of topical interest with immense potential in the field of nanophotonics, high performance nanophosphors and other functional elements (Holmes et al 2000; Bockrath et al 2001; Huang et al 2001; Li and Xia 2003; Xia et al 2003). On the other hand, rare-earth (RE) oxide based nanostructures are found to be promising candidates in the field of high throughput luminescent devices, catalysis and other functional devices based on their excellent electronic, optical and physicochemical responses arising from $4 f$ electrons (Tang et al 2003). Not surprisingly, all these properties could be largely influenced by their chemical composition, crystal structure, shape and dimensionality (Xu et al 2009).

1-D nanostructures can be developed by several methods, such as chemical and physical vapour processes (Yu et al

*Author for correspondence (best@tezu.ernet.in)
2004), laser ablation (Duan and Lieber 2000), solution phase (Trentler et al 1995), arc discharge (Choi et al 2000), hydrothermal treatment (Du et al 2001, 2003; Dhananjaya et al 2012a, b), vapour-phase transport process (Wu and Yang 2000) and template-based method (Huang et al 2000). Above all, hydrothermal synthesis technique has a unique advantage as it is capable of providing high purity and high yield, apart from homogeneously grown anisotropic nanostructures. Besides, hydrothermal technique does not use any catalyst and template. Production of 1-D gadolinium oxides and hydroxides by hydrothermal method have been reported by many authors. Gadolinium oxide $\left(\mathrm{Gd}_{2} \mathrm{O}_{3}\right)$ exists in three different crystallographic forms: hexagonal, monoclinic and cubic (Bai et al 2009). Owing to its thermal and chemical stabilities, narrow emission (Gai et al 2011) and having a wide direct bandgap of $5.3 \mathrm{eV}$ (Singh et al 2004), $\mathrm{Gd}_{2} \mathrm{O}_{3}$ is believed to be a promising host for a number of applications. It can be an useful candidate in the deployment of waveguide devices (Guo et al 2004), high dielectric constant components and other such devices. On the other hand, elongated RE oxides have recently been applied to drug delivery and competitive immunoassays (Liu et al 2012). However, transforming bulk $\mathrm{Gd}_{2} \mathrm{O}_{3}$ powder into its hydroxide product and later to 1 -D nanorods by subsequent dehydration is rarely found 
in the literature. To be specific, obtaining elongated RE nanorods from the bulk powder, short synthesis time along with a catalyst free approach are the main features of the present work.

Herein, we demonstrate production of 1-D $\mathrm{Gd}_{2} \mathrm{O}_{3}$ nanorods via a user-friendly and cost-effective hydrothermal route, without using any sort of catalyst or template. The effect of $\mathrm{pH}$ was discussed in the light of growth mechanism of the nanorods. Characteristic properties with respect to structural, optical emission and magnetic responses of the synthesized nanorods are discussed.

\section{Experimental}

\subsection{Synthesis of $\mathrm{Gd}_{2} \mathrm{O}_{3}$ nanorod powder}

A simple hydrothermal procedure was carried out to produce quality $\mathrm{Gd}_{2} \mathrm{O}_{3}$ nanorods (Dhananjaya et al 2011). In a typical procedure, $0.28 \mathrm{~g}$ of bulk $\mathrm{Gd}_{2} \mathrm{O}_{3}$ (Otto, 99.9\% purity) was first dispersed in $35 \mathrm{~mL}$ distilled water and dilute $\mathrm{HNO}_{3}$ was added dropwise until a clear sol was obtained. $\mathrm{pH}$ was then adjusted to $9 \cdot 2,10 \cdot 8$ and $13 \cdot 3$ by adding several drops of $5 \mathrm{M} \mathrm{NaOH}$ solution under vigorous stirring. The mixture was then transferred to a teflon-lined stainless steel autoclave and subjected to oven heating at $\sim 140{ }^{\circ} \mathrm{C}$ for $24 \mathrm{~h}$. A white solid product of $\mathrm{Gd}(\mathrm{OH})_{3}$ was then collected by filtering the precursor using a Whatman filter ${ }^{\circledR}$. The product was washed several times, first with distilled water and then with ethanol, followed by oven-drying at $80{ }^{\circ} \mathrm{C}$. Finally, the hydroxide powder was annealed at $600{ }^{\circ} \mathrm{C}$, for $3 \mathrm{~h}$ so as to facilitate spontaneous decomposition of $\mathrm{Gd}(\mathrm{OH})_{3}$ and consequently, dehydration to yield $\mathrm{Gd}_{2} \mathrm{O}_{3}$ nanopowder. The flow chart of hydrothermally synthesized $\mathrm{Gd}_{2} \mathrm{O}_{3}$ nanorods is shown in figure 1.

$$
\begin{aligned}
& \text { Acidification: } \\
& \mathrm{Gd}_{2} \mathrm{O}_{3} \text { (bulk) }+6 \mathrm{HNO}_{3} \rightarrow 2 \mathrm{Gd}\left(\mathrm{NO}_{3}\right)_{3}+3 \mathrm{H}_{2} \mathrm{O} \text {, } \\
& \text { Hydrolysis: } \\
& \mathrm{Gd}\left(\mathrm{NO}_{3}\right)_{3}+3 \mathrm{NaOH} \rightarrow \mathrm{Gd}(\mathrm{OH})_{3}+3 \mathrm{NaNO}_{3} \text {, } \\
& \text { Dehydration: } \\
& \text { 2Gd(OH) })_{3} \rightarrow \mathrm{Gd}_{2} \mathrm{O}_{3} \text { (nanopowder) }+3 \mathrm{H}_{2} \mathrm{O} .
\end{aligned}
$$

\subsection{Characterization techniques}

The crystallographic information was revealed by a Rigaku miniFlex X-ray diffractometer (XRD) that uses a CuK $\alpha$ source $(\lambda=1.54 \AA)$. The diffraction angle was varied in the range of $20-60^{\circ}$ and with a step angle of $0 \cdot 05^{\circ}$. Transmission electron microscopy (TEM) images were recorded by a JEOL JEM 2100 machine, operating at an accelerating voltage of $200 \mathrm{kV}$. Thermogravimetric analysis (TGA) was performed on the hydroxide product
$\mathrm{Gd}(\mathrm{OH})_{3}$ of the sample (prepared at $\mathrm{pH}$ 10.8) using a TGA-50 Shimadzu thermogravimetric analyser. The optical absorption study was performed by a UV-Visible spectrophotometer (UV 2450, Shimadzu), while photoluminescence excitation and emission responses were assessed by a Perkin-Elmer LS 55 spectrophotometer. Finally, Raman measurements were recorded by using a Renishaw In-Via Raman spectrometer (Renishaw, Wottonunder-Edge, UK) equipped with an $\mathrm{Ar}^{+}$laser of $\lambda=$ $514.5 \mathrm{~nm}$, used as the excitation source. The spectrum was obtained with a measured resolution of $0.3 \mathrm{~cm}^{-1}$. Lastly, magnetic hysteresis measurement was carried out on a particular specimen (prepared at $\mathrm{pH}=10 \cdot 8$ ) at room temperature using a MPMS SQUID VSM (Quantum Design, USA).

\section{Results and discussion}

\subsection{Structural and morphological features along with growth mechanism}

XRD patterns of the as-synthesized $\mathrm{Gd}(\mathrm{OH})_{3}$ prepared at $\mathrm{pH}=10 \cdot 8$ and $\mathrm{Gd}_{2} \mathrm{O}_{3}$ nanorod-products prepared at $\mathrm{pH}=$ $9 \cdot 2,10 \cdot 8$ and $13 \cdot 3$ are depicted in figure 2(a). The hydroxide product exhibited hexagonal crystal structure with observable diffraction planes indexed as ( $\left.\begin{array}{lll}1 & 1 & 1\end{array}\right),\left(\begin{array}{lll}1 & 0 & 1\end{array}\right)$, (2 0 1) and (0 0 2) (JCPDS no. 83-2037) (Thongtem et al 2010). After annealing at $600{ }^{\circ} \mathrm{C}$, prominent, but apparently broadened diffraction peaks corresponding to (2 2 2), (4 0 0 $)$ ), (4 $\left.4 \begin{array}{ll}4 & 0\end{array}\right)$ and (6 2 2 $)$ crystallographic planes suggest that nanocrystallites would crystallize into a cubic phase (JCPDS no. 86-2477) (Dhananjaya et al 2011). No peak due either to hydroxides, impurities or byproducts was detected. With symbols having their usual meanings and using the diffraction formula relevant to cubic phase: $d_{h k l}=\left(h^{2}+k^{2}+l^{2} / a^{2}\right)^{1 / 2}=\lambda / 2 \sin \theta$, for a first-order diffraction, the crystal cell parameters $(a=b=c)$ could be estimated. The lattice parameters of the samples synthesized at $\mathrm{pH}$ of $9 \cdot 2,10.8$ and $13 \cdot 3$, were estimated to be $10 \cdot 80$, 10.83 and $10 \cdot 79 \AA$, respectively. Earlier, a cubic-phase cell parameter of $10 \cdot 81 \AA$ was predicted for $\mathrm{Gd}_{2} \mathrm{O}_{3}$ system (Ningthousam et al 2009). The average crystallite size $(d)$ and microstrain $(\eta)$ can also be calculated from the popular Williamson-Hall $(\mathrm{W}-\mathrm{H})$ expression

$$
\beta \cos \theta=0.9 \lambda / d+4 \eta \sin \theta,
$$

which represents the equation of a straight line. The average crystallite size was found to be $\sim 5.19,6.46$ and $6.09 \mathrm{~nm}$ for $\mathrm{pH} 9.2,10.8$ and 13.3 cases, respectively along with a negative microstrain value of the order $\sim 10^{-3}$ (table 1). A negative strain could be due to the relaxed nature of the nanocrystallites present in the nanorods.

HRTEM images of the nanorod samples, prepared at $\mathrm{pH}$ $10 \cdot 8$ and $13 \cdot 3$, are shown in figures 2(b) and (c). As for $\mathrm{pH} 10 \cdot 8$, a number of nanorods with an average diameter $\sim 19 \mathrm{~nm}$ and length ranging from 76 to $115 \mathrm{~nm}$ are evident 


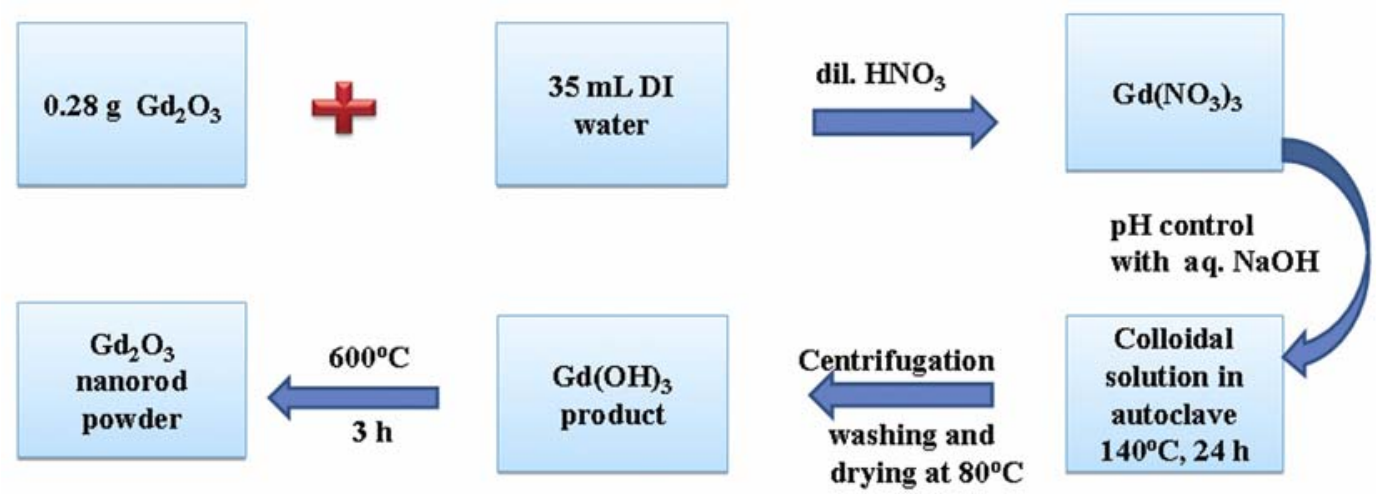

Figure 1. Block diagram of hydrothermally synthesized $\mathrm{Gd}_{2} \mathrm{O}_{3}$ nanorods.
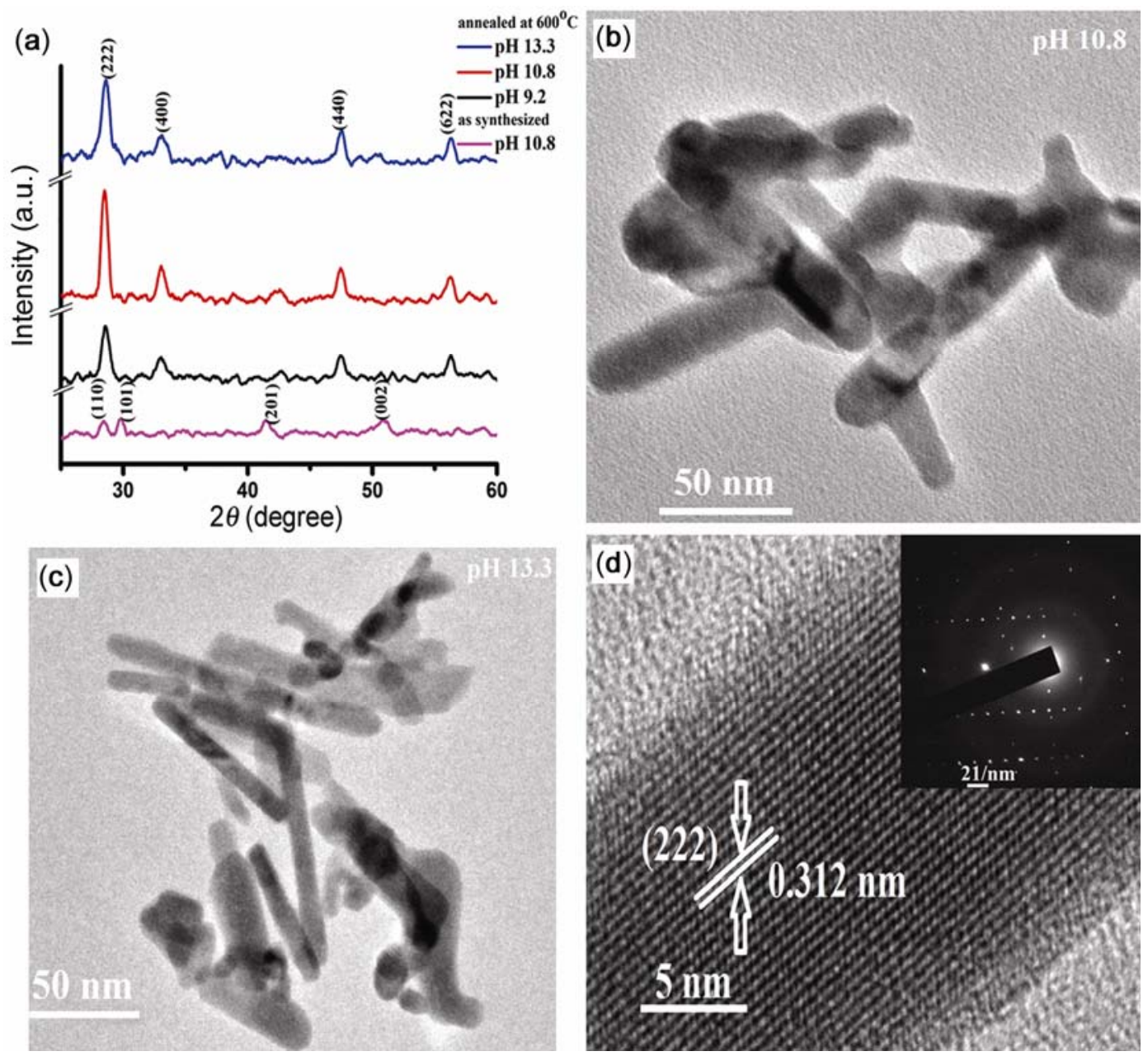

Figure 2. (a) XRD patterns of as-synthesized $\mathrm{Gd}(\mathrm{OH})_{3}$ product ( $\mathrm{pH} 10 \cdot 8$ ) and $\mathrm{Gd}_{2} \mathrm{O}_{3}$ nanorods derived from precursors of different $\mathrm{pH},(\mathbf{b})$ and (c) represent HRTEM images of nanorod samples synthesized at $\mathrm{pH} 10 \cdot 8$ and 13.3, respectively. Magnified image of an isolated $\mathrm{Gd}_{2} \mathrm{O}_{3}$ nanorod (derived from $\mathrm{pH} 10.8$ precursor) with crystal lattice fringes depicted in (d). The SAED pattern is being highlighted in the inset of $(\mathbf{d})$.

in the micrograph (figure 2b). The rods resemble the structure of 'unfold fingers', with smoothened surfaces but without a branching effect. For any nanorod, the diameter at the middle of the rod was much larger than at the extreme-edge. This is ascribed to the spontaneous decom- position of gadolinium hydroxide into its oxide product followed by subsequent assimilation of crystallites along (2 2 2) plane and instant termination at the end. Also, all $\mathrm{Gd}_{2} \mathrm{O}_{3}$ nanorods were not found in the same focal plane and that is why we observed a reasonable difference in 
Table 1. Average crystallite size (nm), lattice parameter $(\mathrm{nm})$, microstrain and dimension of nanorods (nm) of as-synthesized $\mathrm{Gd}_{2} \mathrm{O}_{3}$ nanorod systems.

\begin{tabular}{|c|c|c|c|c|c|c|}
\hline \multirow{2}{*}{$\begin{array}{l}\text { Nanorods synthesized } \\
\text { from precursors }\end{array}$} & \multirow{2}{*}{$\begin{array}{l}\text { Avg. crystallite } \\
\text { size }(d)(n m)\end{array}$} & \multirow{2}{*}{$\begin{array}{l}\text { Lattice parameter } \\
(a=b=c)(\mathrm{nm})\end{array}$} & \multirow{2}{*}{$\begin{array}{l}\text { Microstrain } \\
\left(\times 10^{-3}\right)\end{array}$} & \multicolumn{3}{|c|}{ Dimension of nanorods } \\
\hline & & & & Length (nm) & Diameter (nm) & Aspect ratio \\
\hline pH $9 \cdot 2$ & $5 \cdot 19$ & $1 \cdot 080$ & $-12 \cdot 69$ & - & - & - \\
\hline pH $10 \cdot 8$ & $6 \cdot 46$ & $1 \cdot 083$ & $-8 \cdot 93$ & 75-115 & 19 & $\sim 4$ \\
\hline pH $13 \cdot 3$ & $6 \cdot 09$ & $1 \cdot 079$ & $-8 \cdot 54$ & 60-128 & 7 & $\sim 8.5$ \\
\hline
\end{tabular}

the image contrast of many off-aligned rods. In case of sample synthesized from a precursor of $\mathrm{pH} 13 \cdot 3$, the range of average nanorod length was found to be wider, i.e. 60-128 nm, but having a reduced average diameter of $\sim 7.1 \mathrm{~nm}$ (figure 2c). Thus, both shorter and longer rods were achievable at a higher $\mathrm{pH}$. We speculate that, unrestricted preferential growth of nanocrystallites might have led to longer sized rods. But shorter nanorods could have been developed due to obstruction of one nanorod growing in the vicinity of the other or, thermodynamic instability that would keep surface energy of the overall system minimum. Consequently, the diameter of the rods can also be reduced in this case. As diameter is close to the average crystallite dimension (see XRD analysis), we expect that the aligned growth is manifested by just piling up of single crystallites along a preferred direction. Whereas, nanorods derived from a precursor of lower $\mathrm{pH}$ $(10 \cdot 8)$, comprise of several crystallites along a direction normal to the $c$-axis preferential growth, thereby resulting in a larger diameter $(\sim 19 \mathrm{~nm})$ of the rods. Considering the lower limit of the nanorod length, the aspect ratio was estimated to be $\sim 4$ and 8.5 corresponding to $\mathrm{pH}=10 \cdot 8$ and 13.3 cases, respectively. A higher $\mathrm{pH}$ value represents a higher concentration of $\mathrm{OH}^{-}$ions and a higher chemical potential of the precursor solution. A higher chemical potential is necessary for the growth of higher aspect ratio 1-D nanostructures (Yang et al 2007). In contrary to the results of Yang et al (2007) here, we realized that, nanorods with a higher aspect ratio could be obtained from a high $\mathrm{pH}$ precursor, but along with some amount of inhomogeneity. Figure 2(d) shows an enlarged view of the selected region of a nanorod of figure 2(c). A clear lattice fringe pattern suggests that the nanorod specimen is highly crystalline one with an interplanar spacing of $\sim 0.312 \mathrm{~nm}$ which corresponded to the (2 2 2) crystallographic plane of the nanosystem. This is, in fact, consistent to the XRD pattern of the nano- $\mathrm{Gd}_{2} \mathrm{O}_{3}$ specimen derived from $\mathrm{pH}=10 \cdot 8$. The selected area electron diffraction (SAED) pattern of the nanorods (prepared at $\mathrm{pH}$ 10.8) characterizes a number of arrayed bright spots thus indicating highly crystalline nature of the specimen under study.

The growth mechanism of $\mathrm{Gd}_{2} \mathrm{O}_{3}$ nanorods can be illustrated in a block diagram, shown in figure 3. The growth of $\mathrm{Gd}_{2} \mathrm{O}_{3}$ nanorods was believed to be governed by a solution-solid phase process (Wang et al 2004). In the hydrothermal process, several drops of aq. $\mathrm{NaOH}$ were added to adjust $\mathrm{pH}$ of the solution. According to $\mathrm{Xu}$ et al (2009), the growth morphology is neither governed by a catalyst nor directed by any template $\mathrm{Xu}$ et al 2009). On adding aq. $\mathrm{NaOH}$, the $\mathrm{Gd}^{3+}$ ions tend to interact with $\mathrm{OH}^{-}$ions formed from aq. $\mathrm{NaOH}$. On adding $\mathrm{NaOH}$, a gentle white solution is formed which comprised of colloidal particles. The colloids, while partially dissolved in water results in a metastable supersaturated solution under hydrothermal environment. On cooling back to room temperature, a large number of seed particles of $\mathrm{Gd}(\mathrm{OH})_{3}$ were formed through homogeneous nucleation process. It is the seed which becomes the basis of 1-D elongated nanostructures formed as a result of coherent re-crystallization process. Accordingly, the whole supersaturated solution is recrystallized to yield highly crystalline rod-shaped structures (Xu et al 2009). A high temperature sintering step is needed to facilitate instant dehydration thereby resulting in the desired product of $\mathrm{Gd}_{2} \mathrm{O}_{3}$ nanorods.

Thermogravimetric analysis (TGA) was carried out to assess the progressive weight loss of $\mathrm{Gd}(\mathrm{OH})_{3}$ within a range of temperature $\left(27-600^{\circ} \mathrm{C}\right)$. Typically, TGA curve in figure 4 characterizes four successive, uneven steps. The first step, observable within $27-250{ }^{\circ} \mathrm{C}$ experiences a weight loss of $2 \cdot 1 \%$ and is due to the (partial) dehydration of adsorbed $\mathrm{H}_{2} \mathrm{O}$ in $\mathrm{Gd}(\mathrm{OH})_{3}$. The second step was in the range of $250-335^{\circ} \mathrm{C}$ and having a loss of $\sim 8.02 \%$, whereas third step was between 335 and $440{ }^{\circ} \mathrm{C}$ and with a weight loss of $\sim 1.8 \%$. The second and third steps, ranging from 250 to $440{ }^{\circ} \mathrm{C}$, collectively represent decomposition of $\mathrm{Gd}(\mathrm{OH})_{3}$ to the intermediate product of $\mathrm{GdOOH}$, prior to the complete oxide product. The transformation of intermediate $\mathrm{GdOOH}$ to final $\mathrm{Gd}_{2} \mathrm{O}_{3}$ product is obtained in the fourth step $\left(440-594{ }^{\circ} \mathrm{C}\right)$ and with a weight loss of $1.7 \%$. The total weight loss is $\sim 13.62 \%$, which is comparable to an earlier prediction ( $13.03 \%)$ for complete decomposition of $\mathrm{Gd}(\mathrm{OH})_{3}$ to form $\mathrm{Gd}_{2} \mathrm{O}_{3}$ (Xu et al 2009).

\subsection{Optical absorption, emission and phononic features}

UV-Visible absorption spectroscopy is the most common but an important spectroscopy tool which is capable of revealing electronic transitions of a particular valence 

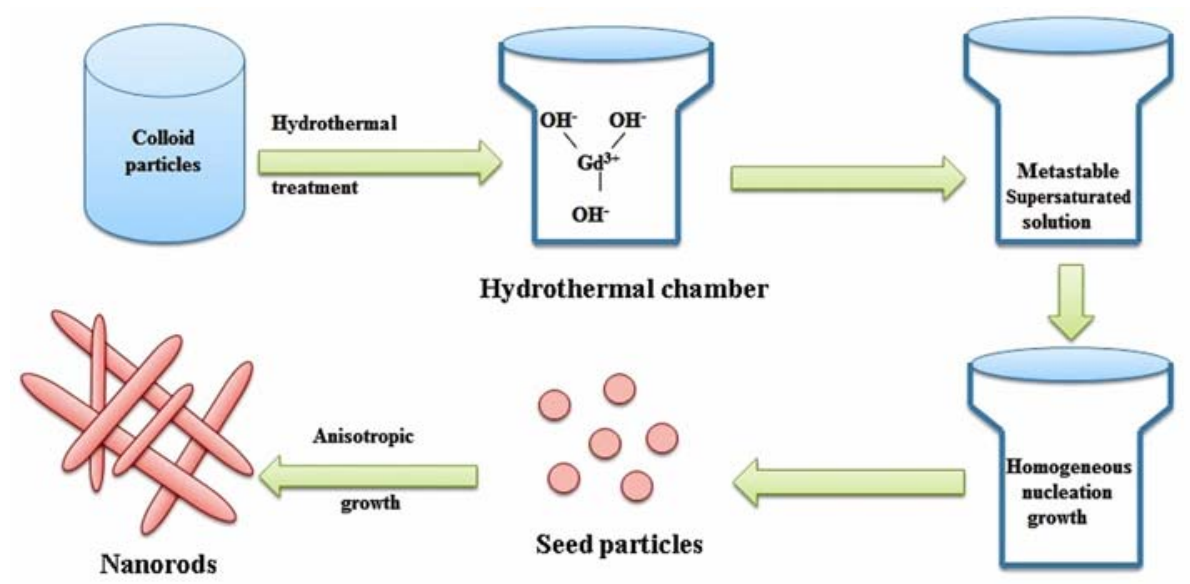

Figure 3. A scheme of growth mechanism illustrating formation of $\mathrm{Gd}_{2} \mathrm{O}_{3}$ nanorods.

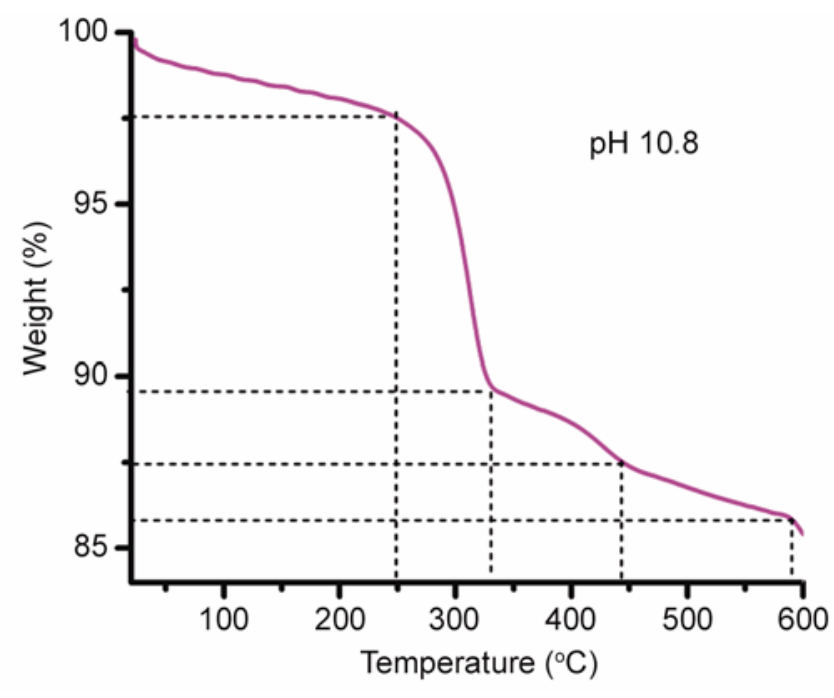

Figure 4. Thermogravimetric analysis of unsintered $\mathrm{Gd}(\mathrm{OH})_{3}$

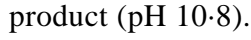

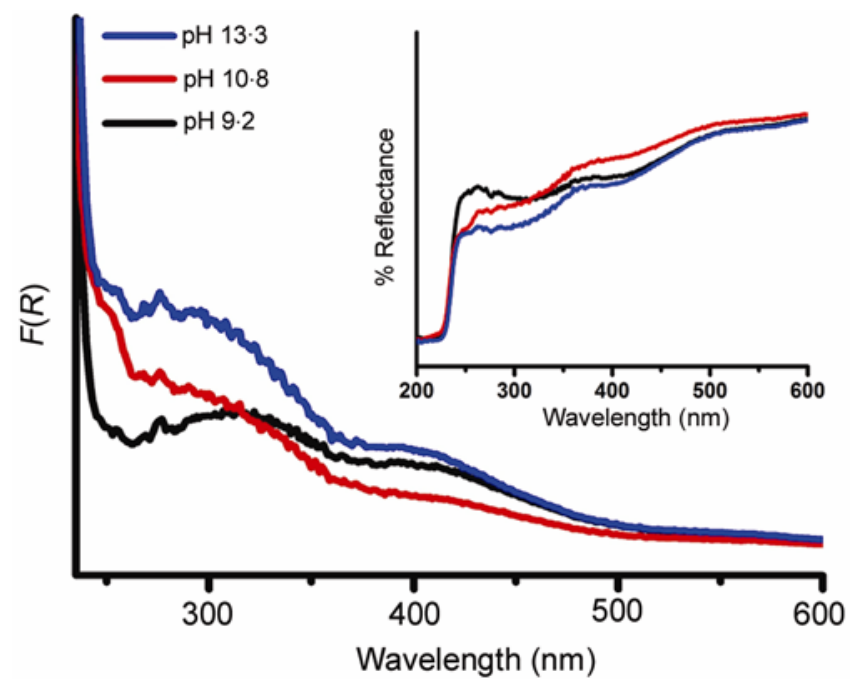

Figure 5. Kubelka-Munk plot representing $F(R)$ as absorbance (inset shows actual reflectance spectra). state. Either absorbance or reflectance (where absorption is not strong) can be employed to determine such transitions. The absorption spectra as obtained from the reflectance data of our nanorod samples are shown in figure 5. The reflectance spectra being shown as the inset of figure 5. For conversion, we have used popular Kubelka-Munk equation

$$
F(R)=(1-R)^{2} / 2 R,
$$

where $R$ represents reflectance and $F(R)$ the corresponding absorbance (Han et al 2007). $\mathrm{Gd}^{3+}$ has [Xe] $4 f^{7}$ configuration, which means it has half-filled shell having a ${ }^{8} S_{7 / 2}$ ground state. The energy absorption in $\mathrm{Gd}$ is mediated via transition of $4 f$ electrons to $5 d$ level and then reorganization of $4 f$ electrons into various multiplets. The seven electrons in $4 f$ orbitals of Gd have as many as 3432 multiplets and the ground state is ${ }^{8} S_{7 / 2}$ (Rahman et al 2011; Hazarika and Mohanta 2013). Among these multiplets, the low lying multiplet above the ground state is ${ }^{6} P_{7 / 2}$. Essentially, two absorption peaks were observed in the spectra. The absorption peak positioned at $\sim 274 \mathrm{~nm}$ is typical for $\mathrm{Gd}_{2} \mathrm{O}_{3}$ nanorods and is attributed to ${ }^{8} S_{7 / 2} \rightarrow{ }^{6} I_{7 / 2}$ transition (Rahman et al 2011). The peak positioned at $\sim 398 \mathrm{~nm}$, may be attributed to recombination of delocalized electrons close to the conduction band with a single charged state of surface oxygen vacancy as per Wang's proposition (Hu et al 2007; Zhang et al 2009). The peak at $\sim 274 \mathrm{~nm}$ has a strong absorption feature, particularly for the nanorod specimen derived at a higher $\mathrm{pH}$ value.

Photoluminescence spectroscopy is a powerful technique to understand the nature of radiative transitions and defect related emission response of a material system. Figure 6(a) shows room temperature photoluminescence excitation (PLE) spectra of $\mathrm{pH} 10.8 \mathrm{Gd}_{2} \mathrm{O}_{3}$ nanorods monitored at an emission wavelength of $\sim 480 \mathrm{~nm}$. The peaks, in PLE spectra, often represent absorption characteristics of the system under study. Basically, $\mathrm{Gd}^{3+}$ has a simpler absorption spectrum in comparison to the other 

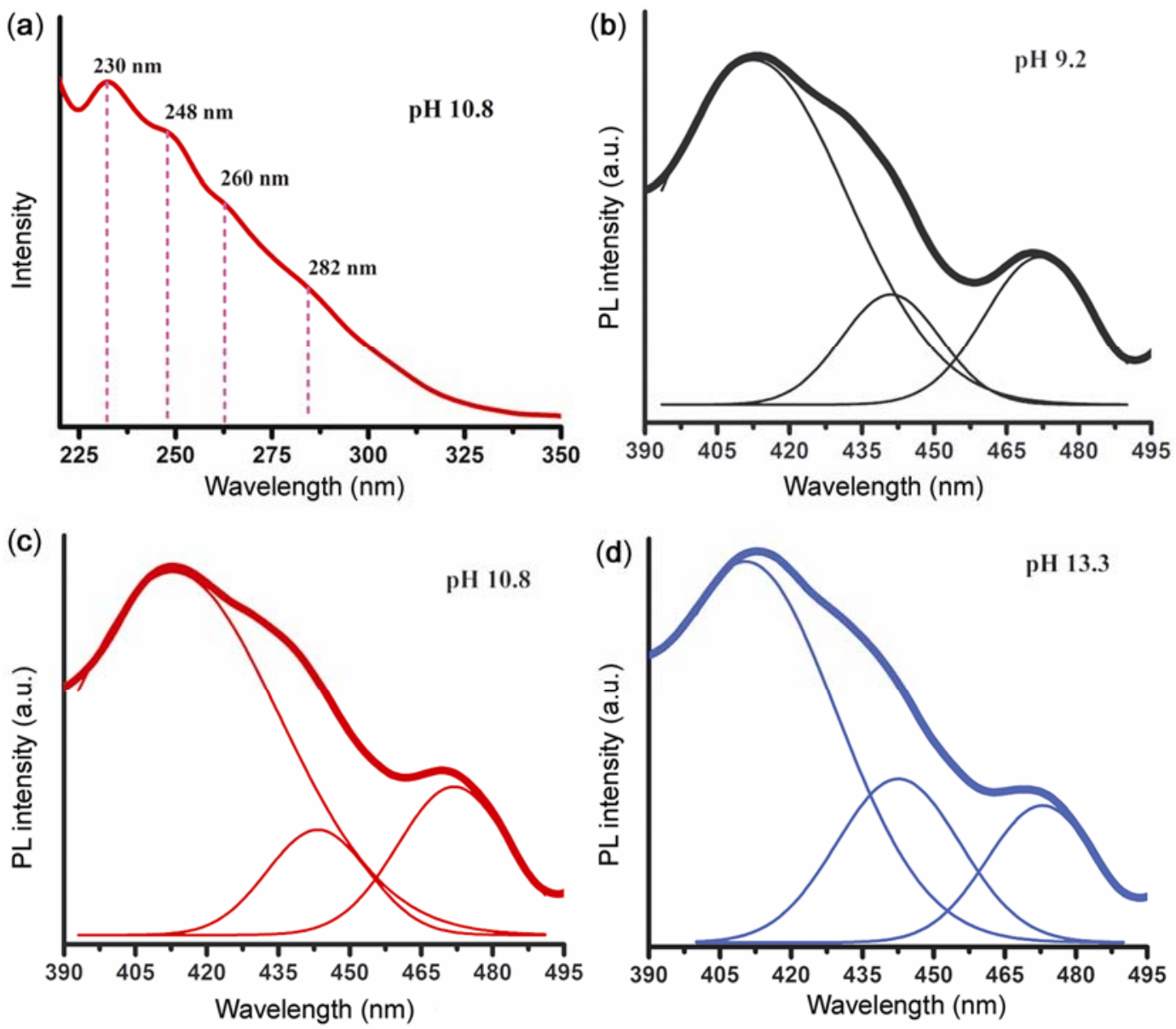

Figure 6. Room temperature (a) PL excitation spectrum of nanorod sample (pH 10.8) considering $\lambda_{\mathrm{em}}=480 \mathrm{~nm}$ and PL emission spectra (with deconvolution) corresponding to $\lambda_{\mathrm{ex}}=300 \mathrm{~nm}$, obtained for all three samples derived at (b) $\mathrm{pH} 9 \cdot 2$, (c) $\mathrm{pH} 10 \cdot 8$ and (d) $\mathrm{pH} 13 \cdot 3$.

trivalent RE ions. Note that, the ground state energy level for $\mathrm{Gd}^{3+}$ is ${ }^{8} S_{7 / 2}$ level. As can be found, the excitation spectra have clearly revealed four important peaks, positioned at $\sim 230,248,260$ and $282 \mathrm{~nm}$. The $230 \mathrm{~nm}$ emission peak is attributed to the excitation band of $\mathrm{Gd}_{2} \mathrm{O}_{3}$ host (Pang et al 2003). The peak at $\sim 248 \mathrm{~nm}$ is ascribed to one of the transitions corresponding to ${ }^{8} S_{7 / 2} \rightarrow{ }^{6} I_{j / 2}$ multiplets, where $j=9,12,13,15$ and 17 (Mukherjee et al 2008). The band observed at $\sim 260 \mathrm{~nm}$ is assigned to ${ }^{8} S_{7 / 2} \rightarrow{ }^{6} D_{9 / 2}$ transition (Kripal and Mishra 2010). The peak at $\sim 282 \mathrm{~nm}$ is attributed to ${ }^{8} S_{7 / 2} \rightarrow{ }^{6} I_{7 / 2}$ transition (Mukherjee et al 2008). On the other hand, room temperature emission spectra of $\mathrm{Gd}_{2} \mathrm{O}_{3}$ nanorods, excited at a wavelength of $\sim 300 \mathrm{~nm}$ are shown in figures 6(b-d) representing characteristic features of the nanorods synthesized at a $\mathrm{pH}$ of $9 \cdot 2,10 \cdot 8$ and $13 \cdot 3$, respectively. The asymmetrically broadened spectra signify the superimposition of several peaks of different origin. The deconvolution of each of the spectra through multiple Gaussian fitting could help extracting independent peaks, which are presented in figures 6(b-d). All the samples have showed three prominent peaks, at $\sim 413,442$ and $472 \mathrm{~nm}$. The band to band emission was not observable due to the improved defect related emission response. The peak maxima at $\sim 413,442$ and $472 \mathrm{~nm}$ could be attributed to surface defects of different Schottky and Frenkel types (Dhananjaya et al 2012a, b). The normalized intensity ratio of $\sim 413$ to $472 \mathrm{~nm}$ peak increases with increasing $\mathrm{pH}$ value of the desired precursor. In oxide systems, typically oxygen vacancies and interstitials are vastly prevalent and the manifestation of such defects, contribute significantly to the modified photoluminescence response.

Raman spectroscopy is a versatile but non-destructive tool to explore vibrational, rotational and other low frequency modes in the systems under study. The microRaman spectrum of the nanorod sample synthesized at a $\mathrm{pH}$ of $10 \cdot 8$, is highlighted in figure 7 . Apparently, three peaks were observed, which were characterized by Raman shifts located at $\sim 314,359$ and $446 \mathrm{~cm}^{-1}$. The peak at $\sim 314 \mathrm{~cm}^{-1}$ is identified as the mixed modes of $F_{\mathrm{g}}+E_{\mathrm{g}}$ and the one at $\sim 446 \mathrm{~cm}^{-1}$ as an independent $F_{\mathrm{g}}$ mode (Dilawar et al 2008). Most importantly, the prominent peak at $\sim 359 \mathrm{~cm}^{-1}$ is assigned to $F_{\mathrm{g}}+A_{\mathrm{g}}$ modes, which mainly corresponds to the cubic phase of $\mathrm{Gd}_{2} \mathrm{O}_{3}$. 


\subsection{Magnetic response of $\mathrm{Gd}_{2} \mathrm{O}_{3}$ nanorod system}

In order to assess magnetic behaviour of the synthesized product, $\mathrm{Gd}_{2} \mathrm{O}_{3}$ nanorod sample (prepared at a $\mathrm{pH} 10 \cdot 8$ ) was assessed by a SQUID magnetometer. It is known that, magnetic response changes with the $\mathrm{pH}$ value of the precursor solution. In particular, saturation magnetization value $\left(M_{\mathrm{s}}\right)$ varies with the particle dimension that depends largely on $\mathrm{pH}$ of the precursor. In this context, modified magnetic features due to an increased fraction of superparamagnetic particles was observed in an earlier work (Thakur et al 2009). Although magnetic measurements were not performed on all the samples, it is

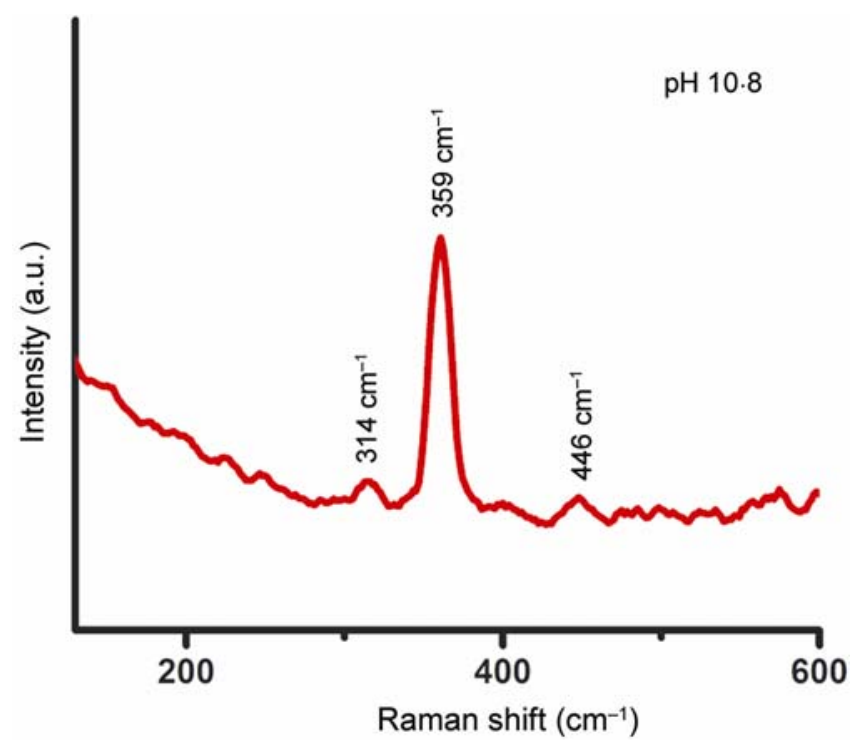

Figure 7. Raman spectrum of $\mathrm{Gd}_{2} \mathrm{O}_{3}$ nanorod sample synthesized at $\mathrm{pH} 10 \cdot 8$.

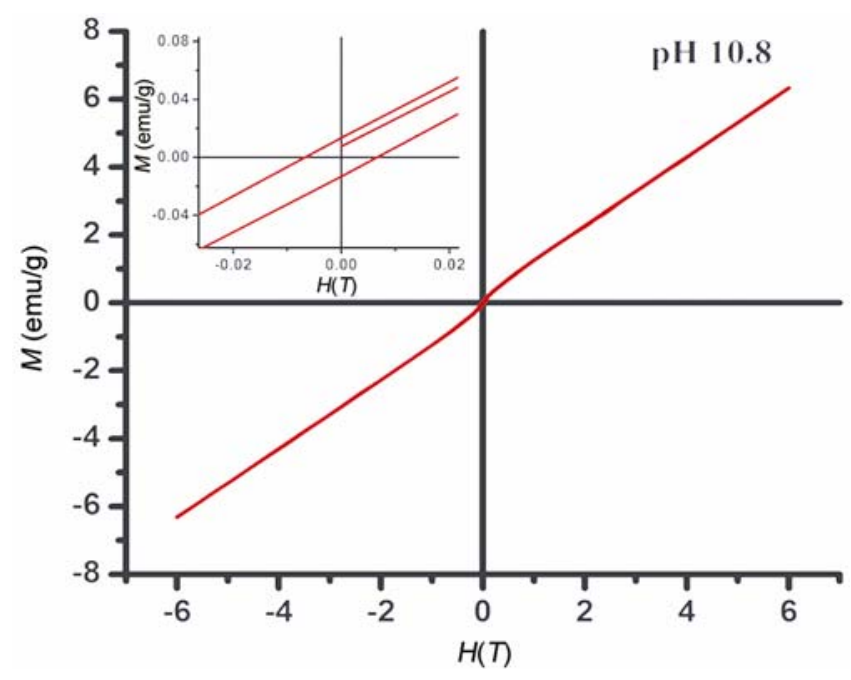

Figure 8. Room temperature $M \sim H$ plot of $\mathrm{Gd}_{2} \mathrm{O}_{3}$ nanorods synthesized at $\mathrm{pH} 10 \cdot 8$. Inset shows enlarged view of plot near zero-field. likely that, $M_{\mathrm{s}}$ value may vary with the aspect ratio of the nanorods (and thus with $\mathrm{pH}$ of the sol). As a means of general assessment (if the system is magnetic or not), magnetic measurement was performed on a wellcharacterized sample $(\mathrm{pH}=10 \cdot 8)$ the results of which are as discussed below.

Figure 8 depicts response of magnetization vs magnetic field $(M-H)$, measured at room temperature (300 K). The magnetization feature was recorded when the applied field was varied in the range -6 to $6 \mathrm{~T}$. Since no clear hysteresis was observed, we speculate that the sample is likely to be superparamagnetic. Even the saturation magnetization could not be predicted exactly. The paramagnetic nature of the nanorods containing $\mathrm{Gd}^{3+}$ centres is due to the presence of half-filled $f$ orbitals with seven electrons (Mukherjee et al 2008). Note that characteristic paramagnetic feature of a system is caused by a number of magnetic dipole moments summing up to zero in the absence of an applied field. But the net moment becomes non-zero when the system is subjected to a field. However, superparamagnetism arises when thermal energy is strong enough to orient individual moments randomly. As far as superparamagnetism is concerned, in the past, most of the studies were devoted mostly to iron based nanostructures. We anticipate that, the paramagnetism in $\mathrm{Gd}_{2} \mathrm{O}_{3}$ nanorods has arisen from non-interacting localized nature of the magnetic moments. The magnetic properties of $\mathrm{Gd}^{3+}$ ions come from seven unpaired inner $4 f$ electrons, which are closely bound to the nucleus and effectively shielded by the outer closed shell electrons $5 s^{2} 5 p^{6}$ from the crystal field. Among various paramagnetic $\mathrm{RE}$ ions, $\mathrm{Gd}^{3+}$ ions possess a high magnetic moment due to isotropic electronic ground state ${ }^{8} S_{7 / 2}$. In order to explore further, we zoomed in $M \sim H$ response near zero field. Remarkably, $M$ value was found to experience a non-zero value in the absence of the field $(H=0)$. This suggests that the investigated sample cannot represent an idealistic superparamagnetic system. The coercive field $\left(H_{\mathrm{c}}\right)$ and remanence $\left(M_{\mathrm{r}}\right)$ were found to be $0.0066 \mathrm{~T}$ and $0.0136 \mathrm{emu} / \mathrm{g}$, respectively. A low coercive field indicates that magnetic moments can be reversed even by changing the direction of a weak applied field. In other words, order of the moments is lost at a higher magnetic field and considering a small remanence magnitude, the $\mathrm{Gd}_{2} \mathrm{O}_{3}$ nanorod sample under study behaved closely to a superparamagnetic system.

\section{Conclusions}

We have demonstrated production and characterization of cubic phase $\mathrm{Gd}_{2} \mathrm{O}_{3}$ nanorods, synthesized by a surfactant free hydrothermal method. XRD studies on $\mathrm{Gd}_{2} \mathrm{O}_{3}$ systems have revealed cubic phase of the as-synthesized nanorods while unsintered $\mathrm{Gd}(\mathrm{OH})_{3}$ product showed a hexagonal phase. HRTEM analyses have revealed a remarkable distribution of nanoscale rods, with aspect 
ratio nearly doubled when the precursor used was changed from $\mathrm{pH}=10 \cdot 8$ to $13 \cdot 3$. Moreover, the evidence of a clear lattice fringe pattern of the rods and ordered bright spots as observable from SAED, suggests that the samples were highly crystalline in nature. TGA analysis on the hydroxide product has revealed the total weight loss $\sim 13.62 \%$ up to a temperature of $\sim 600{ }^{\circ} \mathrm{C}$. The photoluminescence response was found to be manifested by various kinds of defects such as Schottky and Frenkel types. The prominent peak in the Raman spectra attributed to the cubic phase of the nanorods. The magnetic measurements have revealed non-ideal superparamagnetic nature of the $\mathrm{Gd}_{2} \mathrm{O}_{3}$ nanorods at room temperature.

\section{Acknowledgements}

We acknowledge UGC, New Delhi, for financial support received under major project scheme no. 37-367/2009 (SR). We would like to offer sincere thanks to the Department of Chemical Sciences, TU, for extending TGA facility. We also thank SAIF, NEHU, Shillong, for extending HRTEM facility and IIT Kharagpur, for magnetic measurements.

\section{References}

Bai L, Liu J, Li X, Jiang S, Xiao W, Li Y, Tang L, Zhang Y and Zhang D 2009 J. Appl. Phys. 106073507

Bockrath M, Liang W, Bozovic D, Hafner J H, Lieber C M, Tinkham M and Park H 2001 Science 291283

Choi Y C et al 2000 Adv. Mater. 12746

Duan X F and Lieber C M 2000 Adv. Mater. 12298

Du G H, Chen Q, Che R C, Yuan Z Y and Peng L M 2001 Appl. Phys. Lett. 793702

Du G H, Chen Q, Han P D and Peng L M 2003 Phys. Rev. B67 035323

Du G and Tendeloo G V 2005 Nanotechnology 16595

Dhananjaya N, Nagabhushana H, Nagabhushana B M, Rudraswamy B, Shivakumara C, Ramesh K P and Chakradhar R P S 2011 Physica B406 1645

Dhananjaya N, Nagabhushana H, Nagabhushana B M, Rudraswamy B, Sharma S C, Sunitha D V, Shivakumara C and Chakradhar R P S 2012a Spectrochim. Acta Part A: Mol. Biomol. Spectr. 96532

Dhananjaya N, Nagabhushana H, Nagabhushana B M, Rudraswamy B, Shivakumara C and Chakradhar R P S 2012b Bull. Mater. Sci. 35519

Dilawar N, Varandani D, Mehrotra S, Poswal H K, Sharma S M and Bandyopadhyay A K 2008 Nanotechnology 19115703
Gai S, Yang P, Wang D, Li C, Niu N, He F and Li X 2011 Cryst. Eng. Commun. 135480

Guo H, Yang X, Xiao T, Zhang W, Lou L and Mugnier J 2004 Appl. Surf. Sci. 230215

Han T Y, Wu C F and Hsieh C T 2007 J. Vac. Sci. Technol. B25 430

Holmes J D, Johnston K P, Doty R C and Korgel B A 2000 Science $\mathbf{2 8 7} 1471$

Hu C G, Liu H, Dong W T, Zhang Y Y, Bao G, Lao C S and Wang Z L 2007 Adv. Mater. 19470

Huang M H, Choudrey A and Yang P 2000 Chem. Commun. 12 1063

Huang Y, Duan X, Wei Q and Lieber C M 2001 Science 291 630

Hazarika S and Mohanta D 2013 Eur. Phys. J. Appl. Phys. 62 30401

Kripal R and Mishra I 2010 Physica B405 425

Li D and Xia Y 2003 Nano Lett. 3555

Liu Z, Liu X, Yuan Q, Dong K, Jiang L, Li Z, Ren J and Qu X 2012 J. Mater. Chem. 2214982

Mukherjee S, Dasgupta P and Jana P K 2008 J. Phys. D: Appl. Phys. 411

Ningthoujam R S, Shukl R, Vatsa R K, Duppel V, Kienle L and Tyagi A K 2009 J. Appl. Phys. 105084304

Pang M L, Lin J, Fu J, Xing R B, Luo C X and Han Y C 2003 Opt. Mater. 23547

Rahman A T M, Vasilev K and Majewski P 2011 J. Colloid. Interf. Sci. 354592

Singh M P, Thakur C S, Shalini K, Banerjee S, Bhat N and Shivashankar S A 2004 J. Appl. Phys. 965631

Tang Q, Liu Z, Li S, Zhang S, Liu X and Qian Y 2003 J. Cryst. Growth 259208

Thakur S, Katyal S C and Singh M 2009 J. Magn. Magn. Mater. 3211

Thongtem T, Phuruangrat A, Ham D J, Lee J S and Thongtem S 2010 Cryst. Eng. Commun. 122962

Trentler T J, Hickman K M, Goel S C, Viano A M, Gibbons P C and Buhro W E 1995 Science 2701791

Wang G, Wang Z, Zhang Y, Fei G and Zhang L 2004 Nanotechnology 151307

Wang Z, Quan Z and Lin J 2007 Inorg. Chem. 465237

Wu Y and Yang P 2000 Chem. Mater. 12605

Xia Y, Yang P, Sun Y, Wu Y, Mayers B, Gates B, Yin Y, Kim $\mathrm{F}$ and Yan H 2003 Adv. Mater. 15353

Xu Z, Yang J, Hou Z, Li C, Zhang C, Huang S and Lin J 2009 Mater. Res. Bull. 441850

Yu J, Yu J C, Ho W, Wu L and Wang X C 2004 J. Am. Chem. Soc. 1263422

Yang J, Li C, Cheng Z, Zhang X, Quan Z, Zhang C and Lin J 2007 J. Phys. Chem. C111 18148

Zhang N, Ran Y, Zhou L, Gao G, Shi R, Qiu G and Liu X 2009 Mater. Chem. Phys. 114160 